\title{
Investigation of unstable feline diabetes mellitus
}

Danièlle Gunn-Moore and Nicki Reed

\section{Introduction}

Diabetes mellitus is defined as a disorder caused by a relative or absolute lack of insulin. It is a common endocrine disease of cats (see Chapter 13). Despite being likened to type 2 diabetes mellitus of humans, the majority of cats require insulin therapy together with dietary management to control hyperglycaemia and increase the chance of diabetic remission. Although adequate glycaemic control is attained in many cats, a variety of factors can result in diabetic instability (Figure 23.1). These can generally be categorized into:

- Management issues

- Inappropriate type and dose of insulin

- Misinterpretation of blood glucose curves

- Fluctuating insulin requirements

- Insulin resistance.

- Failure to adhere to consistent regime

- Variable timing of injections

- Variable timing of feeding

- Variable diet

- Switching types of diet

- Excess food intake

- Too little food

- Changes in caloric requirements not considered

- Inactive insulin

- Out of date

- Incompletely mixed

- Vigorously shaken

- Incorrectly stored

- Poor injection technique

- Inappropriate syringe type

- Variable doses of insulin drawn up

- Dilution of insulin

- Consistent injection in same site

- Poor subcutaneous absorption

- Inappropriate insulin dose

- Underdosing

- Overdosing resulting in Somogyi overswing

- Fluctuating insulin requirements

- Short duration of insulin activity

- Insulin antibodies

- Poor owner monitoring

- Stress hyperglycaemia

- Concurrent disease

- Diabetogenic medications, e.g. corticosteroids instability in cats.

\section{Management issues}

Management issues include such things as changes in diet or exercise, poor owner compliance, and inappropriate monitoring. It is most common for these problems to arise in the early stages of stabilization. They may be fairly easy to identify and correct after detailed communication with the owner.

\section{Incorrect storage and handling of insulin and poor injection technique}

Insulin may be ineffective if it is out of date, incompletely mixed, damaged from vigorous shaking or poorly stored. Although it is not strictly necessary to refrigerate insulin, this does avoid inactivation caused by increases in ambient temperature or direct sunlight. In addition, insulin will bind to the rubber stopper of the dispensing bottle if it is stored upside down or on its side, thereby resulting in decreased insulin activity within the injection.

It is important to use the correct syringe for the insulin concentration (40 or $100 \mathrm{IU} / \mathrm{ml}$ ). Incorrect doses can be administered through mismatching; it is possible to recalculate the volume of insulin to be administered when using mismatched syringes, but such a step adds an unnecessary complication, increasing the risk of inappropriate dosing.

Some owners may be unable to draw up the correct insulin dose accurately because they have arthritic hands or poor vision. In such cases, the dose administered may frequently change, resulting in significant under- or overdosing at each injection time. Overdosing can lead to insulin-induced hyperglycaemia (Somogyi overswing) (see below). Even for those adept at injecting, accurate drawing up of the small doses of insulin usually required to treat cats can be difficult to achieve consistently. Diluting insulin in order to achieve more accurate dosing may have been recommended, but such dilution can itself be associated with unpredictable results.

Although subcutaneous injections are relatively simple, poor injection technique may be a complicating factor in diabetic instability. This may be particularly important in longhaired cats or those with very dense coats. In such cases, owners may initially benefit from injecting into an area that has been clipped so that they can visualize the technique until greater experience is gained.

Adherence to a consistent daily regime is of vital importance in maximizing the chances of good 
glycaemic control. Problems can arise if, for example, insulin is given at different times each day. There may then be prolonged periods when insulin activity has waned (injection interval too long) or where insulin doses are piggybacked on to each other (injection interval too short).

\section{Other management changes}

Consistency in the feeding regimen is also important and problems may arise if variable quantities or types of food are being offered. Some owners may persist in giving extra food at weekends, switching between wet food on some days and dry on others, giving ad libitum meals on some days and set meals on others, or feeding treats that contain large amounts of soluble sugars. Careful questioning is often required to elucidate the problem as it is more typically children, or occasionally neighbours, that offer the additional food.

There may, however, be occasions when significant changes in energy requirements arise. These include changes in environmental temperature and/ or exercise regime. Owners of diabetic cats that previously hunted and exercised outside may find it easier to keep their cat indoors. Equally, the introduction of a new kitten may entice a diabetic cat to play. In such cases, a change in energy intake may be required, as significant changes in bodyweight (either weight gain or weight loss) can result in significant changes to insulin requirements.

\section{Poor owner monitoring}

Good owner observation is important in determining the degree of glycaemic control in a diabetic cat. Ideally, owners should regularly monitor their diabetic cat for changes in demeanour, appetite, thirst, urination, bodyweight, body condition score and muscle tone, coat condition, mobility and ability to jump. This is easier for some owners than for others. Adequate information may not be available if a number of different people are involved in the cat's care or if the cat spends a great deal of time away from the house or urinates outside. Where significant changes have occurred and the owner has not noticed them, the cat may be presented to the veterinary practice with severely unstable diabetes mellitus, with a greater risk of the development of diabetic ketoacidosis.

Recognition of hypoglycaemia is one of the most important owner considerations. Hypoglycaemic cats rarely exhibit polyphagia but may show a sudden desire to hide, be excessively quiet, weak or lethargic, tremble, become ataxic, collapse or even lose consciousness. Unfortunately, in most affected cats these signs are often subtle and can easily be missed.

\section{Type and dose of insulin}

Cats can be unpredictable in their response to insulin and no single type of insulin or dosing regime is suitable for all cats. Individual responses to exogenous insulin vary not only from cat to cat but also from day to day in the same cat. Therefore, the recommended doses and dosing frequencies are merely guidelines and must be adjusted appropriately based on response.

In cats, lente insulin's time to peak activity can vary from 2 to 10 hours and duration of action can vary from 6 to 16 hours. However, it is usually considered as having a duration of activity close to, though often less than, 12 hours. This compares to protamine zinc insulin (PZI), which has a time to peak activity of 3-12 hours and a duration of activity that varies from 6 to 24 hours. Glargine insulin has a peak effect at approximately 14 hours and duration of action of approximately 24 hours, but often considerably less. Detemir insulin appears to have a similar activity profile to glargine insulin.

Therefore, twice-daily dosing with lente insulin is usually required for adequate glycaemic control, and unstable diabetes mellitus often results from oncedaily dosing. If once-daily insulin injections are desired then PZI may be used, although there are currently problems with availability of authorized veterinary preparations. Additionally, some cats cannot absorb PZI effectively and its use may be associated with poor glycaemic control.

Selecting too high an initial dose of insulin can be problematic. Some authors recommend a starting dose of $0.5 \mathrm{IU}$ insulin/kg per injection, but this is inappropriately high if the blood glucose concentration is $<20 \mathrm{mmol} / \mathrm{l}$. A more appropriate starting dose in such cases is $0.25 \mathrm{IU}$ insulin/kg per injection.

Frequent dose changes, particularly if of high magnitude, can also result in wide swings in glycaemic control. It takes approximately 3 days for glucose homeostasis to adjust after starting or altering insulin doses and, as a consequence, insulin doses should only be increased in small steps (e.g. by a total of $0.5-1.0 \mathrm{IU}$ per injection) followed by a period of at least 3-5 days before reassessing the cat to monitor changes in glycaemic control.

Insulin may be of porcine or bovine origin, recombinant human insulin or synthetic. Of all the insulins available, none is identical to cat insulin. However, although antibody formation to the administered insulin is a theoretical risk, it has not been shown to be of clinical significance in cats.

\section{Interpreting blood glucose curves}

Blood glucose curves are frequently made for the assessment of unstable diabetic cats. The various different types of blood glucose curves typically found are outlined in Figure 23.2. Blood glucose curves can provide important information on the absolute nadir blood glucose concentration, the precise time of the nadir, the duration of effect of insulin, the presence of a Somogyi overswing and the degree by which there is a glucose-lowering effect or not.

Unfortunately, the use of blood glucose curves can be limited in cats for a number of reasons. These include the stress of hospitalization that can itself result in significant hyperglycaemia, the day-to-day variability of blood glucose curves even within the 

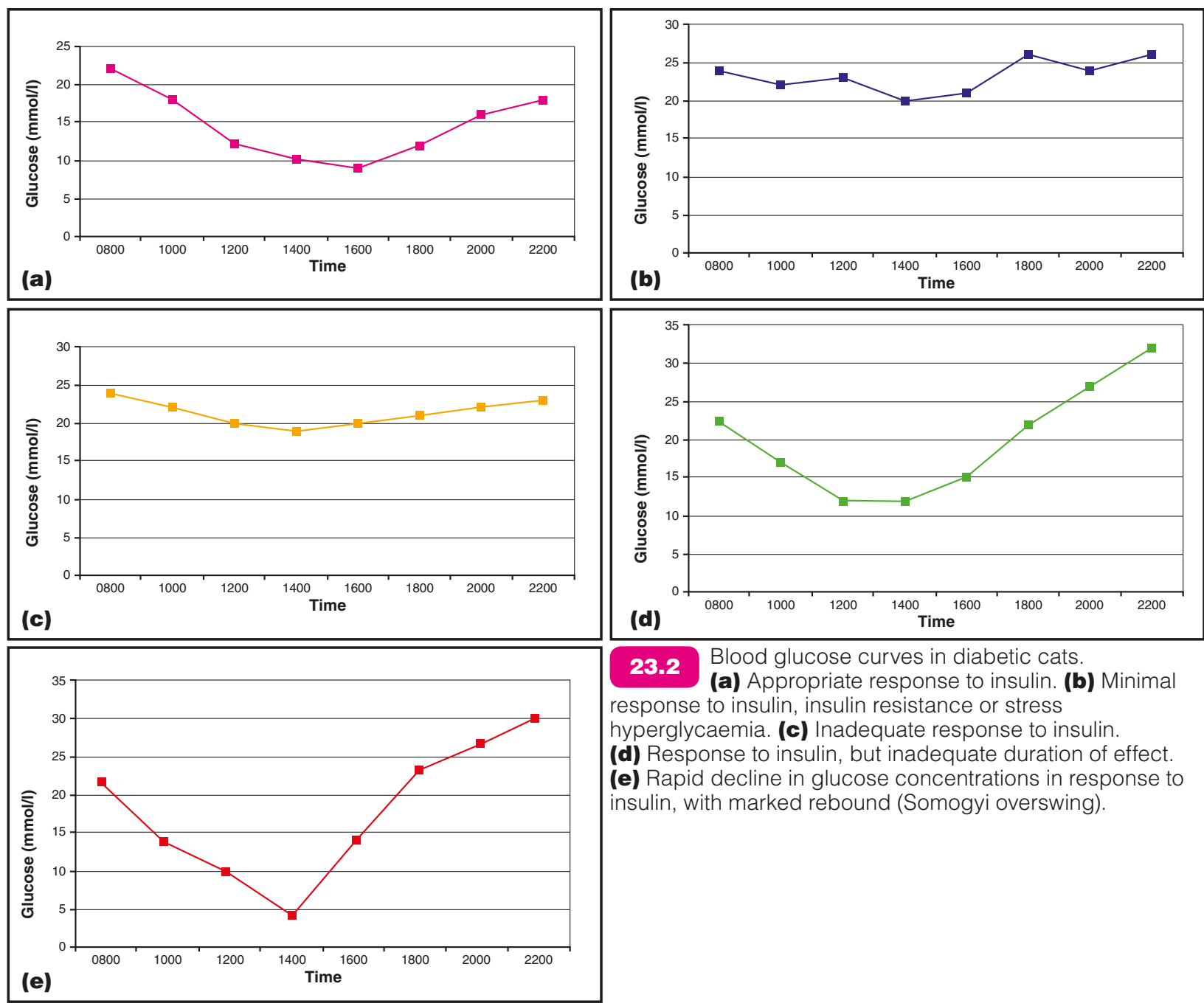

23.2 Blood glucose curves in diabetic cats

(a) Appropriate response to insulin. (b) Minimal response to insulin, insulin resistance or stress

hyperglycaemia. (c) Inadequate response to insulin.

(d) Response to insulin, but inadequate duration of effect.

(e) Rapid decline in glucose concentrations in response to insulin, with marked rebound (Somogyi overswing).

same cat, the possible short duration of hypoglycaemia and the often prolonged duration of any resultant Somogyi overswing.

\section{The effect of stress}

It is meaningless to obtain a blood glucose curve from a cat with stress hyperglycaemia. Unfortunately, stress may not be visibly apparent and can be present even in cats that do not struggle or vocalize. In addition, although stress rarely results in blood glucose concentrations of $>16 \mathrm{mmol} / \mathrm{l}$, this is still sufficient to confuse the interpretation of a blood glucose curve. A stressed cat can be hospitalized, allowed to calm down in its kennel and then reassessed. Other measures to minimize stress include using peripheral ear or paw pad veins (see Chapter 13). Alas, some cats exhibit stress hyperglycaemia every time they are hospitalized. For these cats it may be preferable to train their owners to measure blood glucose concentrations in the home environment. Alternatively, an implanted subcutaneous glucose device and monitor could be used.

\section{Blood glucose measurement}

It is important to ensure that the same type of glucometer is used throughout data collection for each blood glucose curve and for every subsequent blood

glucose curve in an individual cat. Human glucometers tend to read feline glucose concentrations lower than the actual value but may variably underor overestimate values at very high or low concentrations depending on the individual glucometer used. Glucometers validated for feline blood (e.g. Abbott AlphaTRAK) are more likely to give values similar to those from a standard reference laboratory.

\section{Variability}

Whilst blood glucose curves can provide useful information, they can also vary widely from day to day in individual cats. It is therefore important that major changes to treatment are not based on the results from a single blood glucose curve. A blood glucose curve should instead be interpreted in conjunction with the cat's clinical status, fructosamine concentration and the environment in which the curve was obtained. The most important thing to consider is the trend of change, so comparing the current curve with previous blood glucose curves is important.

\section{Somogyi overswing}

The Somogyi overswing describes a normal physiological response to hypoglycaemia induced by excessive insulin administration. This commonly occurs when insulin doses are increased too quickly with 
inadequate monitoring, or if the cat has fluctuating insulin requirements. When blood glucose concentrations reduce to $<3.5 \mathrm{mmol} / \mathrm{l}$ or when they fall precipitously (>10 mmol/l in 1 hour), counter-regulatory hormones such as cortisol, glucagon and adrenaline are secreted, resulting in a rebound hyperglycaemia within a few hours. This hyperglycaemia persists for at least 24 hours in most cases, but can last for up to 72 hours, and occasionally even longer. Clinical signs of hypoglycaemia are rarely seen.

A diagnosis of Somogyi overswing is achieved by demonstrating hypoglycaemia or a rapid fall in blood glucose concentration followed by hyperglycaemia. Unfortunately, the hypoglycaemia can be missed if blood samples are taken less frequently then every hour at peak insulin activity and the subsequent rebound hyperglycaemia and insulin resistance can last for considerably more than 24 hours. A cyclic history of 1-2 days of good glycaemic control followed by several days of poor control should raise particular suspicion for the Somogyi overswing. If considered a possibility, the insulin dose should be reduced by $0.5-1.0 \mathrm{IU}$ or $25-30 \%$ and the cat monitored for the next few days. If originally overdosed, the cat should become more stable; if not, it is likely to become more polydipsic and polyuric.

\section{Fluctuating insulin requirements}

It is not unusual for some cats to have fluctuating insulin requirements, with apparently uncontrolled diabetes mellitus at one time and hypoglycaemia or hyperglycaemia the next. The most common reasons for this are:

- Transient diabetes mellitus (see Chapter 13)

- The development of a concurrent disease that causes variable insulin resistance and later resolves spontaneously, or waxes and wanes.

\section{Insulin resistance}

The majority of diabetic cats can be controlled with insulin doses of approximately $0.4-0.6 \mathrm{IU} / \mathrm{kg}$ administered twice daily. Poor glycaemic control and/or insulin resistance are generally defined by an insulin requirement of $>1.5-2.0 \mathrm{lU} / \mathrm{kg}$. This can be associated with a wide variety of different problems and conditions (Figure 23.3) and can be complex to investigate.

As diabetic cats tend to be older, it is not surprising that there is a risk of concurrent disorders that may affect diabetic stability. The presence of any concurrent disease, especially those involving an inflammatory response or infectious focus, can cause marked insulin resistance. One study found concurrent disease in $22 \%$ of diabetic cats (Crenshaw and Peterson, 1996). Those commonly diagnosed include chronic kidney disease, hyperthyroidism, pancreatitis, urinary tract infection (UTI), acromegaly and hyperadrenocorticism (Goossens et al., 1998; Feldman and Nelson, 2004). While
- Recent weight gain (common)

- Infection, e.g. urinary tract infection (12-14\%) or gingivitis

- Pancreatitis or other forms of pancreatic pathology (acute or chronic pancreatitis 6-50\%; pancreatic adenocarcinoma 19\%; exocrine pancreatic insufficiency $4 \%$ )

- Administration of diabetogenic drugs (9\%), e.g. corticosteroids, progestogens

- Hyperthyroidism (9-29\%)

- Acromegaly $(14-19 \%)$

- Hyperadrenocorticism (14-17\%)

- Kidney disease (15-33\%)

- Liver disease (2-10\%)

- Inflammatory bowel disease (3\%)

- Neoplasia (2-9\%)

- Congestive heart failure (up to $15 \%$ )

- Eosinophilic granuloma complex $(3 \%)$

- Asthma (2\%)

- Immune-mediated disease $(2 \%)$

- FIV-positive (2\%)

23.3 Concurrent disease that may potentially result in insulin resistance in diabetic cats. The

percentages refer to the frequency of occurrence in cats with diabetes mellitus (data from Goossen et al.,1998; Feldman and Nelson, 2004).

these different diseases may occur concurrently, some occur together more frequently and are potentially related to the development of diabetes mellitus or its consequences (pancreatitis, acromegaly). For some of the disorders highlighted in Figure 23.3 (eosinophilic granuloma complex, asthma, neoplasia, immune-mediated disease) the administration of systemic corticosteroids for their management can result in unstable diabetes mellitus rather than the presence of the condition itself.

\section{Obesity}

Any increase in bodyweight is likely to cause significant insulin resistance. Recent weight gain despite poor diabetic control is most commonly due to acromegaly, although it can occasionally be seen with hyperadrenocorticism or excessive feeding.

\section{Infection}

In diabetic cats, infection is most likely to affect the mouth (gingivostomatitis and/or periodontal disease), urinary tract, or skin. Diabetic animals are predisposed to infection because of systemic immunosuppression resulting from decreased peripheral blood supply, impaired humoral immunity and antibody production, abnormal neutrophil chemotaxis and defects in phagocytosis and intracellular processing of infectious agents (Joshi et al., 1999).

\section{Pancreatitis}

Pancreatitis and other forms of pancreatic pathology often result in variable insulin requirements. Pancreatitis may be more common than previously thought, as $>50 \%$ of diabetic cats have evidence of past or current pancreatitis at necropsy. Pancreatitis can occur together with inflammatory bowel disease (IBD) and cholangitis ('triaditis'). Where this occurs, the presence of these conditions can further complicate diabetic stability, particularly if they remain undiagnosed. Chronic pancreatitis can eventually 
lead to significant loss of pancreatic function with possible development of exocrine pancreatic insufficiency (EPI). Unfortunately, pancreatitis can be difficult to diagnose and affected cats may show few or non-specific clinical signs, such as anorexia and lethargy. Measurement of feline pancreatic lipase immunoreactivity (fPLI) is the most sensitive diagnostic test available, but a reference interval fPLI does not exclude pancreatitis. Abdominal ultrasonography and pancreatic biopsy can be useful tools for the diagnosis of pancreatitis. Achieving a diagnosis of pancreatitis is unlikely to alter the treatment regimen for the diabetes mellitus but provides some explanation as to why a cat may have extremely variable insulin requirements.

\section{Hyperthyroidism}

Hyperthyroidism complicates the diagnosis and treatment of diabetes mellitus. The duration of action of exogenous insulin may be shorter than expected in cats with concurrent hyperthyroidism, and any endogenous insulin secretion may be reduced. Unfortunately, the possibility of concurrent hyperthyroidism may be missed in cats with diabetes mellitus as the clinical signs can be similar. In addition, a diagnosis of hyperthyroidism is complicated by the suppressive effect of diabetes mellitus on total thyroxine (T4) concentrations. Circulating fructosamine concentration may also be lowered by hyperthyroidism, suggesting better glycaemic control than is actually present. Evaluation of free T4 concentration may help in the diagnosis of hyperthyroidism.

\section{Acromegaly}

Acromegaly (Chapter 5) is an uncommonly reported, but probably underdiagnosed, condition in cats. It results from overproduction of growth hormone $(\mathrm{GH})$ from a pituitary tumour, typically an adenoma of the pars distalis. In addition to diabetes mellitus, cats with acromegaly usually exhibit enlargement of the head, paws and abdomen and inferior prognathism. Some cats may also develop neurological signs, osteoarthritis, and cardiac and kidney disease. Definitive diagnosis of acromegaly requires demonstration of elevated concentrations of $\mathrm{GH}$ or insulin-like growth factor-1 (IGF-1) together with a pituitary mass, by either computed tomography (CT) or magnetic resonance imaging (MRI). As growth hormone assays and advanced imaging techniques are poorly available, an elevated IGF-1 concentration (especially if >131 $\mathrm{nmol} / \mathrm{I})$, together with clinical signs consistent with acromegaly, are usually considered adequate for a diagnosis. The possibility of underlying acromegaly should be considered in any unstable diabetic cat. A particularly high index of suspicion should be present in any older, large, male diabetic cat that is gaining weight despite the presence of unstable diabetes mellitus.

\section{Hyperadrenocorticism}

Hyperadrenocorticism is a rare but also possibly underdiagnosed disease in cats (see Chapter 17). In approximately $80 \%$ of cases it is caused by adrenocorticotropic hormone (ACTH)-secreting pituitary tumours, most of which are adenomas. In a few cases, hyperadrenocorticism is caused by a functional adrenal tumour, which can be either adenomatous (approximately $50 \%$ of cases) or carcinomatous.

Hyperadrenocorticism is typically seen in middle-aged to older cats (range 4-16 years) with no apparent sex or breed predisposition. The history and clinical signs often include polyuria, polydipsia, polyphagia, weight loss, generalized muscle loss and lethargy, and may include a history of recurrent infections and/or abscesses. Affected cats often have a coat that is in poor condition, with spontaneous alopecia, and fragile thin inelastic skin that bruises easily (fragile skin syndrome). Occasionally, they develop seborrhoea and/or bacterial dermatitis and a pot-bellied appearance that results from weakened abdominal muscles, obesity and, in some cases, hepatomegaly. As cortisol antagonizes insulin, the majority of cases develop diabetes mellitus and are frequently insulin resistant.

In cats, screening tests include the urine cortisol: creatinine ratio (UCCR), low-dose dexamethasone suppression test (LDDST) and the ACTH response test, while differentiation of pituitary from adrenal dependency may involve measurement of endogenous ACTH concentration or diagnostic imaging modalities (adrenal glands by ultrasonography, or $\mathrm{CT} / \mathrm{MRI}$ assessment of pituitary gland). Some cases that mimic hyperadrenocorticism actually have progestogen-secreting adrenal tumours.

\section{Kidney disease}

Kidney disease is commonly seen in cats with diabetes mellitus and in one study was found in approximately one-third of cases (Goossens et al., 1998). Kidney disease is not uncommon in older cats but might also be caused or exacerbated by ascending bacterial infections, diabetic nephropathy or significant hypotension in those cases with diabetic ketoacidosis or severe pancreatitis. As renal function deteriorates, significant insulin resistance may develop. However, the anorexia associated with kidney disease potentially increases the risk of hypoglycaemia, resulting in fluctuating insulin requirements in affected cats. Monitoring of diabetic control becomes more difficult, as the degree of polyuria and polydipsia is obviously affected by renal function.

\section{Liver disease}

Liver disease may occur in diabetic cats. Hepatic lipidosis may develop in unstable or newly diagnosed cats, while cholangitis often occurs concurrently with IBD and/or pancreatitis.

\section{Neoplasia}

Neoplasia anywhere in the body can lead to inflammation and insulin resistance. The tumours seen most commonly in unstable diabetic cats are pancreatic adenocarcinoma, lymphoma and mast cell tumours (Ogilvie et al., 1997). 


\section{Administration of diabetogenic drugs}

Diabetogenic drugs include corticosteroids (dexamethasone is more diabetogenic than equipotent doses of prednisolone) or progestogens such as megestrol, particularly when given at high doses and/or for long courses. Corticosteroids do not require systemic administration to influence diabetic control, as topical eye and ear preparations can be absorbed sufficiently to affect insulin requirements.

\section{Other problems}

A failure of insulin absorption mimics the insulin resistance seen with concurrent disorders. It may be a result of injecting the insulin at the same site every day or injecting the insulin into fat depots. It can also be a feature of long-acting insulin administration. In dogs, insulin antibodies potentially result in insulin resistance. However, such antibodies appear to be rare in cats.

\section{Investigation of instability}

Investigation of diabetic instability can be challenging, time-consuming and expensive. Each category of instability should be considered by obtaining an appropriate history, completing a thorough physical examination and pursuing additional diagnostic tests as considered necessary. A step-by-step approach is outlined in Figure 23.4.

\section{History}

The first important step when investigating problems of diabetic stabilization is to ask the owner detailed questions about their cat's daily management regime, particularly any recent changes. Many problems with stabilization result from poor owner compliance, which in turn often results from poor direction, support and communication from the veterinary team. When investigating an unstable diabetic cat it is necessary to ensure that clients understand the importance of adhering to a consistent regime of insulin administration, diet and exercise. Careful questioning will usually reveal if such problems are possible, but if they are not identified at this point it may be necessary to ask the owner to demonstrate how they mix and inject the insulin, in order to check that this is being done correctly. Any such problems can be easily rectified without the need for further protracted investigations.

If problems of management or insulin administration are eliminated then further questioning may be necessary. Owners need to be asked about changes in their cat's health and behaviour, including ascertaining information on demeanour, appetite, thirst, urination, bodyweight, muscle tone, coat condition, mobility and ability to jump. Questions should focus on those concurrent diseases that can significantly complicate the treatment of diabetes. Cats with unstable diabetes resulting from chronic pancreatitis may have a history that includes episodes of depression, anorexia, vomiting, diarrhoea and/or abdominal pain, and if most of the pancreas has been destroyed these clinical signs may also be accompanied by signs of EPI (i.e. a voracious appetite and large quantities of voluminous fatty faeces). Cats with acromegaly may present with weight gain despite diabetic instability, while those with hyperthyroidism may lose weight despite significant polyphagia.

\section{Physical examination}

Each cat should have a full physical examination, including assessment of bodyweight, calculation of percentage bodyweight change since the previous visit, body condition score, retinal examination and systemic blood pressure measurement.

It is important to differentiate signs of diabetic instability from signs of another underlying or concurrent illness. Signs of diabetic instability include an unkempt coat, hepatomegaly and a plantigrade stance. Cats with UTI may additionally exhibit signs of cystitis such as bladder and/or kidney discomfort, dehydration. The mouth should be carefully checked as severe periodontal disease is a possible reason for insulin resistance. Concurrent pancreatitis may result in dehydration, abdominal discomfort and, in severe cases, jaundice. The neck should be carefully palpated for goitre.

\section{Blood glucose curve}

The next step in the investigation depends on the abnormalities detected from the history and on physical examination. Where significant concomitant disease is suspected, further investigations should include routine clinicopathological analyses (see below). However, where this is not the case and a problem with insulin dose or dosing frequency is suspected, it is more appropriate to obtain a blood glucose curve (see above and Chapter 13). Such curves may provide information on short duration of activity or the possibility of a Somogyi overswing (see Figure 23.2). If either of these problems is identified then the frequency of dosing should be increased or the dose decreased, respectively. If insulin resistance is noted, then other causes should be considered.

\section{Routine clinicopathological analyses}

Each cat should have a full haematology, serum biochemistry (including electrolytes, total T4 concentration and fructosamine concentration), plus full urine analysis with sediment assessment and bacterial culture.

\section{Haematology}

Haematological changes may result from associated systemic infections or severe stress. The most typical changes are a mild non-regenerative anaemia, lymphopenia and either neutropenia or neutrophilia. Acute pancreatitis may present with severe red cell fragility and haemolytic anaemia, and/or sequestration of neutrophils resulting in a degenerate left shift. Severe cases may develop haematological changes consistent with disseminated intravascular coagulation. 


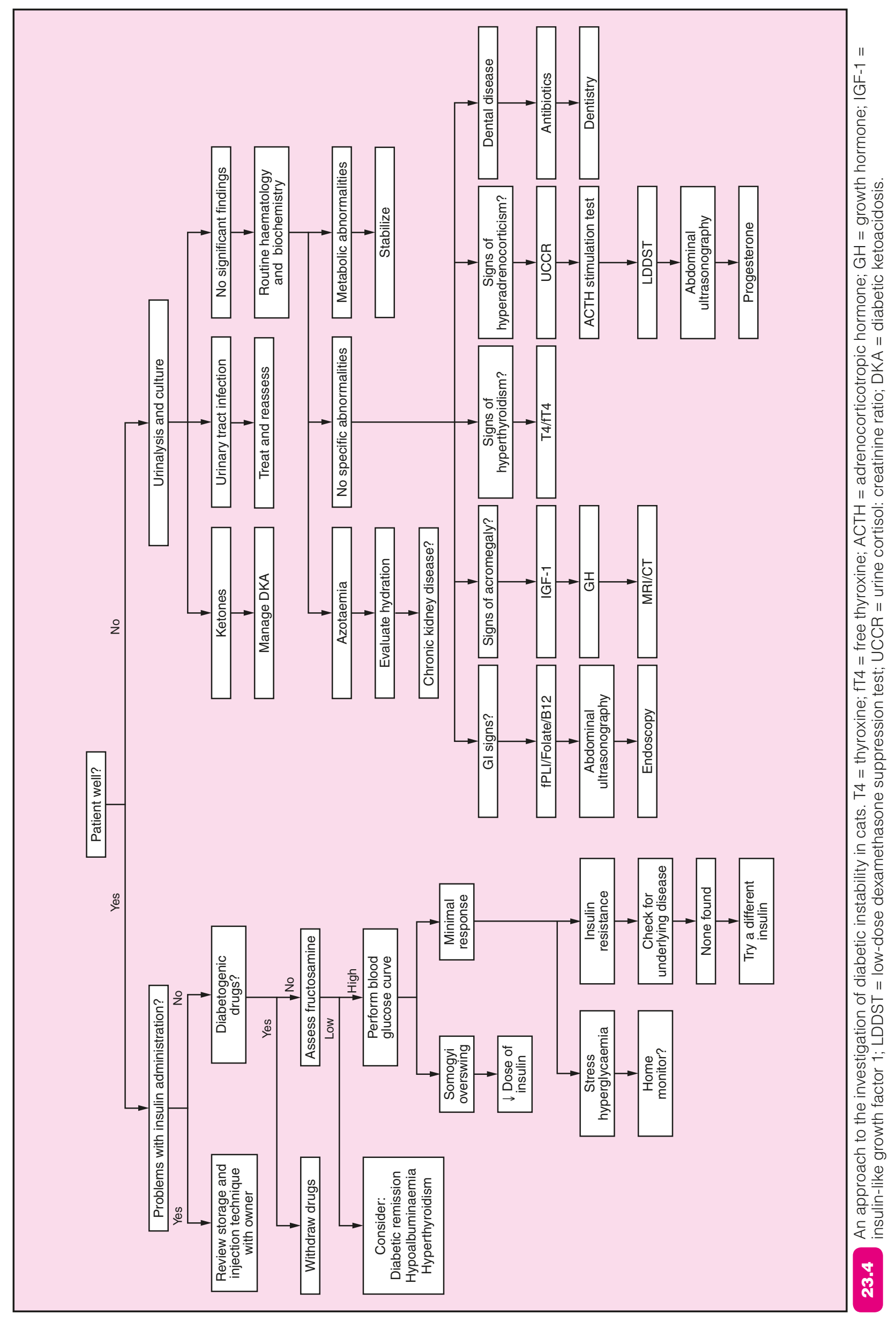




\section{Serum biochemistry}

Serum biochemistry can be helpful in detecting concurrent and complicating disease but changes associated with unstable diabetes mellitus need to be considered. Many cats with diabetes mellitus have mild to moderate increases in serum concentrations of cholesterol and liver enzymes. More severe changes including hyperbilirubinaemia and azotaemia usually indicate the presence of a complicating disorder.

\section{Fructosamine concentration}

Assessing serum fructosamine concentration can be useful, as it gives an indication of how elevated the blood glucose concentration has been for the preceding 1-2 weeks. High concentrations (>500 $\mu \mathrm{mol} / \mathrm{l})$ indicate poor glycaemic control. However, such high values may occur both in insulin resistant states and with insulin overdose if it results in Somogyi overswing. Care is also required as fructosamine can be raised where there has been prolonged stress hyperglycaemia. Additionally, it may not be raised in the initial stages of diabetic instability and can be falsely lowered in some conditions such as hyperthyroidism that increase protein turnover.

\section{Total thyroxine concentration}

Circulating total T4 concentration should be assessed to rule out the presence of concurrent hyperthyroidism. Care should be taken in its interpretation as the presence of diabetes mellitus can result in suppression of a previously mildly elevated total T4 concentration into the reference interval. In the absence of hyperthyroidism, total T4 concentrations would be expected in the lower half of the reference interval in diabetic cats.

\section{Urine analysis}

Urinalysis is essential to assess the presence of ketones and glucosuria as indicators of diabetic instability. However, caution is advised as stress hyperglycaemia can result in glucosuria. The urine specific gravity should be noted and interpreted appropriately, as the presence of $2 \%$ or $4+$ glucosuria will increase the specific gravity by $0.008-$ 0.010 , which may complicate the diagnosis of concurrent kidney disease. Sediment examination will help in the diagnosis of UTIs but some of these cats have a non-active urinary sediment. It is therefore essential to collect a sample by cystocentesis and perform urine culture regardless of the sediment findings.

\section{Further investigation}

Depending on other findings this may involve additional testing as follows:

- $\mathrm{GH}$ and/or IGF-1 and assessment of pituitary size when investigating acromegaly
- UCCR, LDDST, endogenous ACTH concentration, and assessment of adrenal and pituitary size by ultrasonography, CT or MRI when investigating hyperadrenocorticism

- Serum fPLI, fTLI, cobalamin and folate concentrations when investigating pancreatic and/or intestinal pathology

- Survey radiographs of thorax and abdomen for cardiac, respiratory disease or evidence of neoplasia

- Abdominal ultrasonography (paying particular attention to the pancreas, liver, kidneys and adrenal glands)

- Gastrointestinal endoscopy and biopsy for diagnosing IBD

- Hepatic biopsy and/or bile culture to diagnose cholangitis.

More detailed information on the diagnosis and treatment of other endocrine disorders is presented in relevant chapters.

\section{References}

Crenshaw KL and Peterson ME (1996) Pretreatment clinical and laboratory evaluation of cats with diabetes mellitus: 104 cases (1992-1994). Journal of the American Veterinary Medical Association 209, 943-949

Crenshaw KL, Peterson ME, Heeb LA, Moroff SD and Nichols R (1996) Serum fructosamine concentration as an index of glycemia in cats with diabetes mellitus and stress hyperglycemia. Journal of Veterinary Internal Medicine 10, 360-364

Elliott DA, Feldman EC, Koblik PD, Samii VF and Nelson RW (2000) Prevalence of pituitary tumors among diabetic cats with insulin resistance. Journal of the American Veterinary Medical Association 216, 1765-1768

Feldman EC and Nelson RW (2004) Canine diabetes mellitus; Feline diabetes mellitus In: Canine and Feline Endocrinology and Reproduction, 3rd edn, ed. EC Feldman and RW Nelson, pp. 486-538; 539-579. WB Saunders, Philadelphia

Forcada Y, German AJ, Noble PJM et al. (2008) Determination of serum $\mathrm{fPLI}$ concentrations in cats with diabetes mellitus. Journal of Feline Medicine and Surgery 10, 480-487

Goossens MMC, Nelson RW, Feldman EC and Griffey SM (1998) Response to insulin treatment and survival in 104 cats with diabetes mellitus (1985-1995). Journal of Veterinary Internal Medicine 12, 1-6

Joshi N, Caputo GM, Weitekamp MR and Karchmer AW (1999) Infections in patients with diabetes mellitus. New England Journal of Medicine 341, 1906-1912

Koenig A, Drobatz KJ, Beale AB and King LG (2004) Hyperglycemic hyperosmolar syndrome in feline diabetics: 17 cases (19952001). Journal of Veterinary Emerging and Critical Care 14, $30-40$

Kraus MS, Calvert CA, Jacobs GJ and Brown J (1997) Feline diabetes mellitus: a retrospective mortality study of 55 cats (1982-1994) Journal of the American Animal Hospital Association 33,107-111

Little CJL and Gettinby G (2008) Heart failure is common in diabetic cats: findings from a retrospective case-controlled study in firstopinion practice. Journal of Small Animal Practice 49, 17-25

Niessen S (2010) Feline acromegaly: an essential differential diagnosis for the difficult diabetic. Journal of Feline Medicine and Surgery 12, 15-23

Ogilvie GK, Walters L, Salman MD et al. (1997) Alterations in carbohydrate metabolism in dogs with nonhematopoietic malignancies. American Journal of Veterinary Research 58, 277-281

Rand JS (2002) Understanding feline diabetes. Compendium on Continuing Education for the Practicing Veterinarian 24(5), Supplement B, 2-6

Reusch CE, Kley S, Casella M et al. (2006) Measurements of growth hormone and insulin-like growth factor 1 in cats with diabetes mellitus. Veterinary Record 158, 195-200 